\title{
SMART HELMET WEAR FOR DRIVER SAFETY
}

\author{
Ayesha A. Momin ${ }^{1}$, Jyoti M. Aldale ${ }^{2}$, Sajida M. Pinjari ${ }^{3}$, Amruta B. Kale ${ }^{4}$ \\ ${ }^{1}$ B.E Student, Department of Electronics and Telecommunication Engineering, AMGOI, Maharashtra, India \\ ${ }^{2}$ B.E Student, Department of Electronics and Telecommunication Engineering, AMGOI, Maharashtra, India \\ ${ }^{3}$ B.E Student, Department of Electronics and Telecommunication Engineering, AMGOI, Maharashtra, India \\ ${ }^{4}$ Asst.Professor, Department of Electronics and Telecommunication Engineering, AMGOI, Maharashtra, India
}

\begin{abstract}
In today's world road accident is a major problem. Negligent of the driver is the major fact of such accident. There are many rules under the government for the motor drivers for vehicles but many of them disobey the Government rule. We all know the helmet is best safekeeping appliance for driver safety, this system will satisfy the purpose of saving life. This system is consist of sensors like limit switch sensor, alcohol sensor, and accelerometer sensors. Limit switch sensor is used to check helmet is wearing or not, alcohol sensor is used to check alcohol presence in driver's breath. If rider is wearing the helmet \& not drunken then fuel system \& ignition system is started. Accelerometer sensor to measure tilt position of helmet. If the accident is occurred, accident location is traced by using the GPS module and then message is send to family member and nearest hospital by using GSM module.
\end{abstract}

Keywords - Sensors- Limit switch sensor, alcohol sensor, accelerometer sensor, ATmega328 Micro Controller, GPS, GSM Module

\section{INTRODUCTION}

Nowadays the use of two wheeler and four wheeler are increases. As per the report $34.5 \%$ accident deaths occurred on National Highways, while $27.9 \%$ accident death took place on State Highway's. Death of person and head injury is major problem of accident. To reduce this problem our system is used to protect the human life.

Motorcycle helmets are one of the most important consideration of all safety concerns, especially when you consider the current statistics stating that brain injury is one of the most dominant causes of motorcycle facilities. It is good to see that the government have stepped into make it mandatory for the riders. Motorcycle helmets are basically aimed at absorbing the impact of collision to the brain in case of an accident. With this in mind, among the first things that one should be looking for is the material of the helmet. Lightweight, tough and crack resistant fibers and grades of

Plastic have made it into the helmet manufacturing process in a big way. Impact resistance can be gauged from the shape and size of helmets. ****

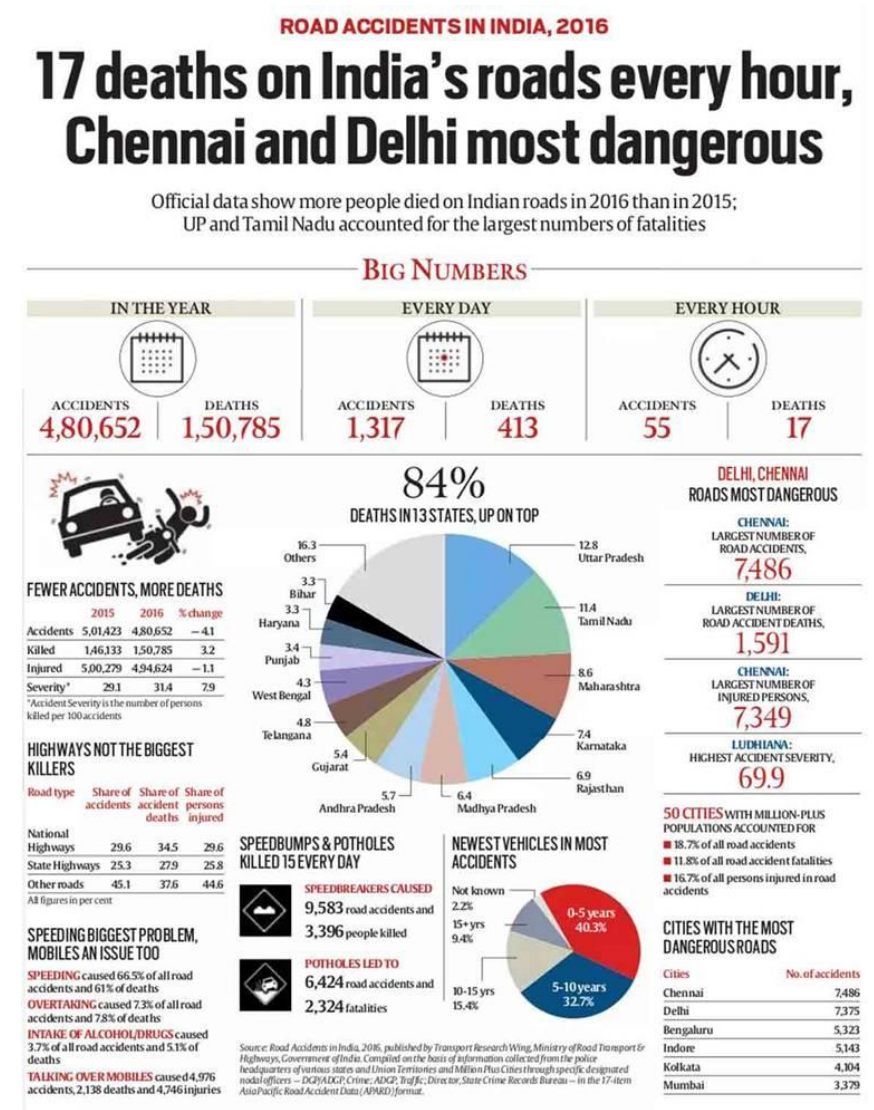

In current situation there is most cases of accidents area by motor bikes. The percentage of those accidents are increased because of the absence of helmet or by the usage of 
alcoholic drinks. To solve this system we will develop an electronic smart helmet system that efficiently checks the wearing of helmet and drunken driving. By implementing this system a safe 2 wheeler journey is possible which would decrease the head and body injuries. Another feature of that system having the ability to detect the accident location and send the location on predefined number using a GPS and GSM system respectively.

\section{PROBLEM ANALYSIS}

Nowadays the use of motorcycle is increases all over world but the use of motorcycle is the major problem to occur the road accident because of the absence of helmet and drunken driver. Due to road accident the head injury and there is possibility of death. This happen because avoiding the use helmet.

\section{PROBLEM FORMULATION}

The system which can be used to avoid the road accident and giving the detection of accident where the accident is going to happened. Using smart helmet technology it is possible to detection of wearing helmet or not by limit switch sensor, rider is drunken or not by alcohol sensor, tilt position measured by accelerometer sensor.

When accident is happened then accident location tracked by GPS module an1d send the message to the predefined number. The system is only started when the rider wear the helmet and rider is not drunken then fuel system and ignition is started of bike.

\section{PROPOSED WORK}

\subsection{Helmet Side Circuit (Transmitter Section)}

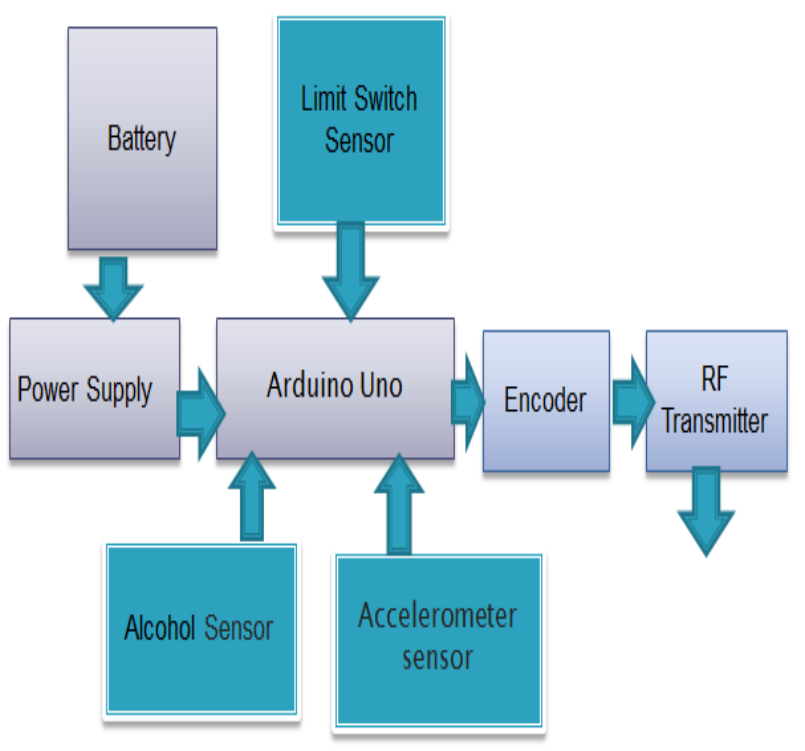

The block diagram of transmitter section is as shown in above figure. Helmet side section is nothing but a transmitter. This transmitter section is consist of RF transmitter, sensors like limit switch sensor to check wearing the helmet or not, alcohol sensor to check the alcohol presences in drivers breath, accelerometer sensor for check movement of helmet, all signal are send to encoder through Arduino. Receives data from these sensors and it gives a digital data corresponding to the output of sensors to the encoder only if the conditions are satisfied. Encoders that are transmitted by a carrier using an RF transmission medium.

\subsection{Bike Side Circuit (Receiver Section)}

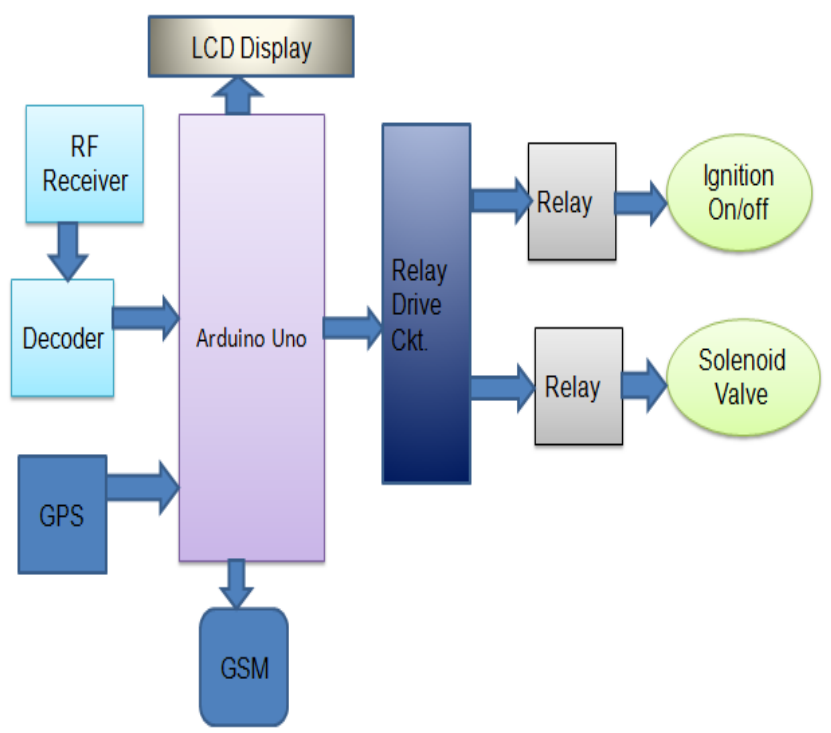

Fig 4.2: Block Diagram of Bike Section

The Bike side circuit mainly consists RF receiver receives the data, they compare the serial input data three times continuously with their local addresses. If no error or unmatched codes are found, the input data codes are decoded and then transferred to the output pins.. Decoder decodes the input and gives data to Arduino only in the address bit of encoder and decoder matches and the relay drive circuit also interfaced which controls the Ignition onoff and also controls Fuel on-off. Normally relay is an electro-magnetic switch which is used in low voltage circuit to switch on and off solenoid valve connected to a $5 \mathrm{v}$ DC supply. Using GPS and GSM module we can detect the exact location of the system and whenever accident is happened that location is send by SMS to the predefined number of family member or a nearest hospital of that area.

Fig: 4.1 Block Diagram of Helmet Side 


\section{EXPERIMENTAL RESULT:}

\subsection{Circuit Diagram of Helmet Side (Transmitter Section)}

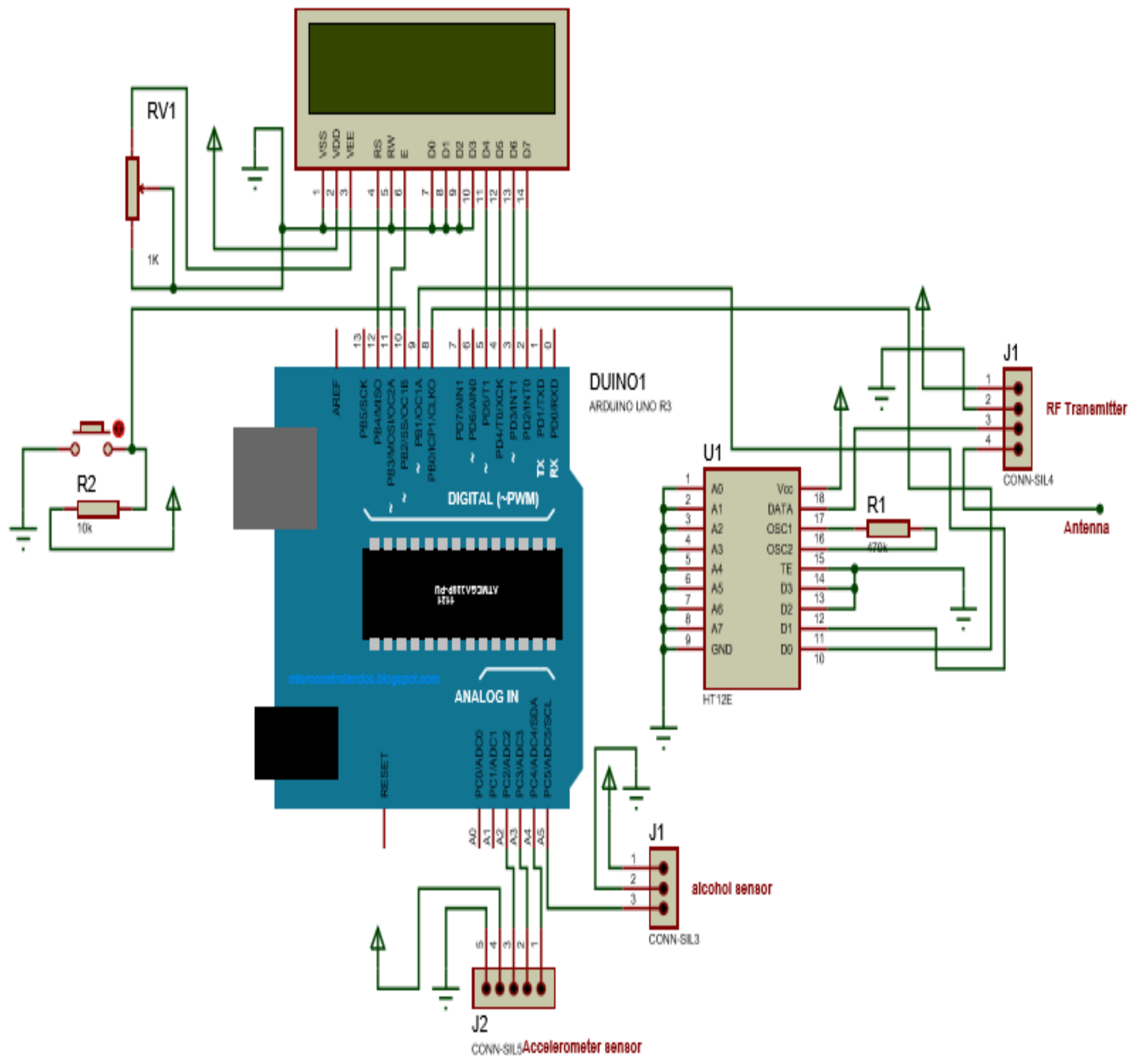

Fig 5.1: Circuit Diagram of Helmet Side 


\subsection{Circuit Diagram of Bike Side (Receiver Section)}

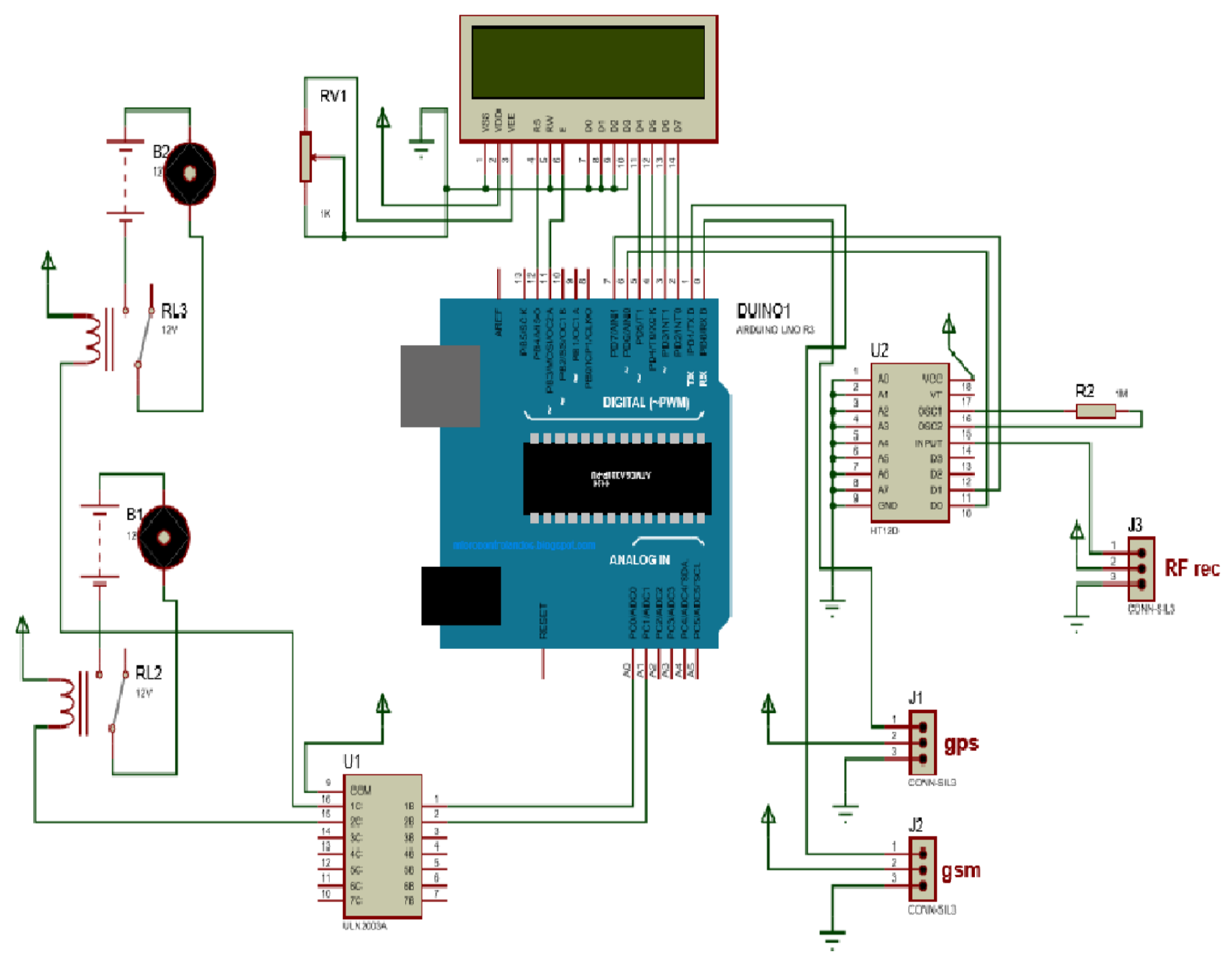

Fig 5.2: Circuit Diagram of Bike Side

\section{CONCLUSION}

The main object of this system to secure and avoid road accident. The communication between bike and helmet section so user have to must wear the helmet and no presence of alcohol then bike will get start and hence the Government rules will follow with this. Location is traced with the help of GPS technology and send the accident location using GSM technology. Thus the Smart Helmet system provide the better security to the bike riders.

\section{REFERENCES}

[1]. Lakshmi Devi P, Bindushree R, Deekshitha N M, Jeevan M, Likhith M P, "Helmet Using GSM And GPS Technology For Accident Detection And Reporting System”, ISSN 2321-8169, In May 2016.

[2]. Devendra Itole, Dnyaneshwar Avatirak , Omkar Kale, Nikhil Deshpande, Aakash Menon "Intelligent Helmet”,IJPTT In March 2015.
[3]. Seokju Lee, Girma Tewolde, Jaerock Kwon, "Design And Implementation Of Vehicle Tracking System Using GPS/GSM/GPRS Technology And Smart Phone Application", IEEE World Forum On Internet Of Things, in March 2014.

[4]. Sudharsana Vijayan, Vineed T Govind Merin Mathews, Simna Surendran, Muhammed Sabah M E,"Alcohol Detection Using Smart Helmet System”ISSN: 0976-1353, in April 2014.

[5]. Abhinav Anand, Kumar Harsh, Kushal Kumar, Sourav Gouthi, "Microcontroller Based Smart Wear For Driver Safety", ISSN 2321-7308, in May-2015

[6]. Ashwinkumar T. Limbanee, Angraj G. Kad, Swapnil N. Jagtap, Komal S. Lonari, Prof. Shiv Sutar,'Smart Helmet to Avoid Road Kill(SHARK)",ISSN 2350-1022,in March 2017.

[7]. K. Rambabu, B.Premalatha, C.Veeranjaneyulu,"An Optimal Driving System by Using Wireless Helmet”.ISSN:2278-7798, in Sept 2013 
[8]. Pushkar Shukla, Shejar Safaya, Shashi Kant, "Human Safety System for two Wheelers-A Microcontroller based Prototype", ISSN 2278-1021, in Aug-2014.

[9]. Manjesh N.,Prof.Sudarshan Raj, "Smart Helmet Using GSM\&GPS Technology for Accident Detection and Reporting System",ISSN 2348-6988,in Dec-2014.

[10]. Abhi Khan, Ravi Mishra," PS-GSM Based Tracking System", ISSN 2231-5381, in 2012 